\title{
Nationwide Survey of Healthcare Services for Autism Spectrum Disorders (ASD) in Italy
}

\author{
Marta Borgi ${ }^{1} \cdot$ Valentina Ambrosio $^{1} \cdot$ Daniele Cordella $^{2} \cdot$ Flavia Chiarotti $^{1} \cdot$ Aldina Venerosi $^{1}$
}

Published online: 23 May 2019

(C) The Author(s) 2019

\begin{abstract}
Objectives ASD is a lifelong multifaceted condition with considerable impact and costs to the single patients, their family and wider society, hence emphasizing the need of tailoring services for ASD subjects and their families. In Italy, recent efforts have been made to harmonize and promote the appropriateness of interventions for ASD people. At present, scattered information on services for people with ASD is available. The aim of this study was to survey the presence and distribution of child and adolescent mental health services (CAMHs) in Italy, devoting a specific focus on their capacity to provide care to ASD patients. Methods CAMHs based on local health services, public and university hospitals, and care/research institutions, as well as private (INHS-accredited) units were invited to participate to present investigation filling in a questionnaire assessing relevant dimensions for public health, namely overall structural capacity and ASD care processes.

Results Heterogeneity of the results among geographical macro-areas in CAMHs provision was found. Specifically, for ASD care, diagnosis and intervention strategies resulted to be unevenly distributed across the Italian territory, and the level of service integration throughout transition into adulthood resulted to be low.

Conclusions Results from this survey contribute to increase the knowledge on services provided in Italy to people with ASD, and suggest potential targets to improve ASD management. The final aim is to ensure that the conditions are in place to achieve the adequate capacity to respond to the needs of individuals affected and their families over time and across the whole country.
\end{abstract}

Keywords Autism spectrum disorders $\cdot$ Child-adolescent mental health services $\cdot$ Systems of care $\cdot$ Italy

Based on national and international estimate of prevalence (Elsabbagh et al. 2012; Narzisi et al. 2018), there are about 600,000 people with Autism Spectrum Disorders (ASD) in Italy, including about 100,000 under the age of 18 years. ASD is a lifelong condition with considerable impact and costs to the individuals, their family, and wider society (Howlin et al. 2013). Direct and indirect (e.g., individual and parental productivity loss), as well as lifetime social economic costs, have been associated with ASD (Buescher et al. 2014). Families of children with autism face higher levels of distress than those of children with other disabilities: psychological disorders are common in the caregivers and appear to be marked with high levels of stress, anxiety, and depression (Lai et al. 2015; Picardi

Aldina Venerosi

aldina.venerosi@iss.it

1 Center for Behavioral Sciences and Mental Health, Istituto Superiore di Sanità, Viale Regina Elena 299, 00161 Rome, Italy

2 Management Control and Information Technology, Istituto Superiore di Sanità, Viale Regina Elena, 299, Roma, Italia et al. 2018; Stewart et al. 2017). Parents also suffer a strong impact in term of time burden, divorce, and loneliness (Chan et al. 2018; Fernandez-Alcantara et al. 2016).

The increasing estimate of ASD prevalence, the high complexity of the disorder and its economic burden, demand for timely interventions addressing a wide array of development and adaptive behavior and support services for the entire family (Lord et al. 2018). However, we still identify gaps between families' needs and the services offered (Hodgetts et al. 2015; Turcotte et al. 2016). A recent survey conducted in Europe showed considerable variation across countries regarding both access to interventions and type of intervention received (Salomone et al. 2016). When the views and experiences of professionals in the UK were explored, different areas in need of improvement were identified, including knowledge and training, clear and open referral pathways into services, and time taken to access first appointments within services (Rogers et al. 2016). Professionals interviewed expressed also dissatisfaction with post-diagnostic provision, especially onward and long-term support options. These findings highlight the need to monitor service provision for children with ASD at 
country level, in order to contrast inequalities in service accessibility and strengthen public health capacities in term of organizational structures, resources, workforce, partnerships, and governance.

In Italy, the institutional agreement among the Ministry of Health and the regions (hereinafter referred to as the Italian ASD Action Plan, IAAP; Conferenza Unificata 2012, 2018), as well as the Italian law on ASD (Law n. 134/2015), identify strategic priorities for ASD health care, with the final aim of promoting harmonized protocols for ASD diagnosis and evidence-based intervention approaches, according to recommendations defined at national and international level (SIGN 2016; NICE 2013; SNLG 2011; NICE 2011).

One of the priority objectives of the IAAP was to increase the knowledge on services provided in Italy to children and adolescents with ASD, in order to ensure that ASD services achieve the adequate capability to meet the needs of individuals and their families across the whole country.

In the Italian National Health System (INHS), neurodevelopmental disorders - including ASD - are in charge of the specific Child and Adolescent Mental Health units (CAMHs), which are based on local health services, public and university hospitals, and care/research institutions (see Fig. 1 for a more comprehensive network of national, regional, and local institutions and services involved in ASD health care). Moreover, INHS-accredited private providers (Art. 26 Law 388/78) are widely distributed across the Italian territory and offer rehabilitation services to people with ASD.
According to priorities outlined by IAAP, the main aims of this study were to provide information on the presence and distribution of CAMHs within the Italian territory, and on their capability to provide care to ASD patients, namely diagnostic and functional assessment, as well as efficient interventions. To this aim, we surveyed public and private (INHS-accredited) CAMHs available nationwide and we analyzed and evaluated ASD care provided by them according to two dimensions, namely structural capacity (i.e., allocation and provision of qualified human resources) and process (i.e., services provided to ASD patients) (Donabedian 1986; Handler et al. 2001). Quantitative comparisons between public and private facilities and among geographical macro-areas were performed. At the same time, we collected information on regional policies concerning ASD, since they represent one of the variables at the supra-system level that can directly or indirectly affect care delivery (Donabedian 1986; Handler et al. 2001).

\section{Method}

\section{Participants}

Regional authorities (contact points) were asked to provide the list of CAMHs present in each regional area (hereafter named "expected CAMHs"). CAMHs based on local health services (Aziende Sanitarie Locali, ASLs), public and university hospitals (Aziende Ospedaliere, AOs; Aziende Ospedaliere
Fig. 1 Network of national, regional, and local institutions and services involved in ASD health care in Italy

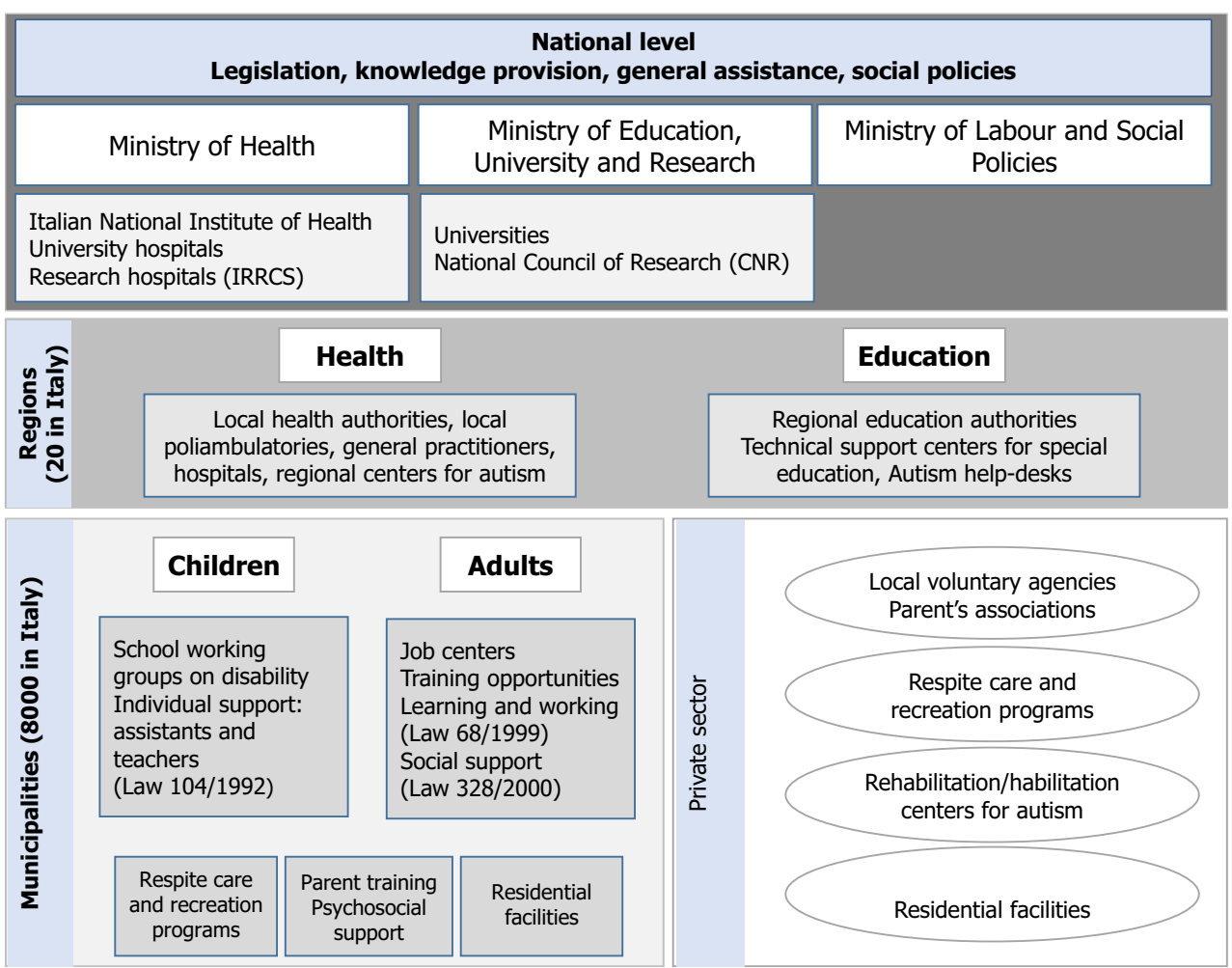


Universitarie, AOUs), and care/research institutions (Istituto di Ricovero e Cura a Carattere Scientifico, IRCCSs), as well as private (INHS-accredited) units, were all considered eligible for the survey. The invitation to participate in the survey was emailed to the listed units in September 2013. After two email contacts non-respondents were contacted again by telephone.

Table 1 shows the number of the expected public and private (INHS-accredited) service providers covering each macro-area, as listed by contact points of regional authorities. Table 1 also shows the average number of target population ( $0-17$ years) for each CAMH. This was computed as the number of children aged $0-17$ years living in the corresponding catchment area (official data provided by ISTAT) divided by the number of expected CAMHs in the same area. Of the 722 expected CAMHs, 518 participated in the survey (Table 1). The overall survey response rate was $72 \%$, computed excluding three regions for which an official list of CAMHs was not provided. Of the 499 surveyed units, 351 were ASLs, 13 were AOs, 12 AOUs, 9 IRCSSs, and 114 were private units.

\section{Procedure}

A questionnaire was developed by researchers of the Italian Institute of Health (Istituto Superiore di Sanità) in collaboration with the Italian Ministry of Health. Specialists in the ASD field, as well as professionals of target services were consulted and contributed to develop the form. To ensure that questions and instructions were clear and unambiguous, the questionnaire underwent a pilot sample of $10 \mathrm{CAMHs}$ and was slightly revised according to participants' suggestions.

Table 1 Number of expected and surveyed CAMHs across the different macro-areas and population burden

\begin{tabular}{llll}
\hline CAMHs & $\begin{array}{l}\text { Number of } \\
\text { expected } \\
\text { CAMHs }\end{array}$ & $\begin{array}{l}\text { Number of } \\
\text { surveyed } \\
\text { CAMHs }\end{array}$ & $\begin{array}{l}\text { Mean population } \\
(0-17 \text { yrs }) \text { per } \\
\text { CAMH* }\end{array}$ \\
\hline
\end{tabular}

$\begin{array}{lrrr}\text { Public } & & & \\ \text { North } & 166 & 153 & 14,393 \\ \text { Center } & 123 & 104 & 15,576 \\ \text { South } & 116 & 60 & 21,202 \\ \text { Islands } & 95 & 87 & 12,152 \\ \text { Italy } & 500 & 404 & 15,942 \\ \text { Private } & & & \\ \text { North } & 41 & 27 & 46,290 \\ \text { Center } & 68 & 36 & 27,258 \\ \text { South } & 63 & 32 & 40,386 \\ \text { Islands } & 50 & 19 & 23,089 \\ \text { Italy } & 222 & 114 & 33,559\end{array}$

*Computed as the number of children aged 0-17 years living in the macro-area (1st January 2012, ISTAT) divided by the number of expected CAMHs in the same macro-area
A Moodle ${ }^{\circledR}$ platform was used to create the online survey. Units were given the options to access the online questionnaire or to fill in the paper version of the form and to send it by mail or email. Telephone support was provided to the respondents. Data were collected from September 2013 to December 2015.

\section{Measures}

The structure of the questionnaire was based on a conceptual framework containing relevant dimensions for public health capacity, namely structural capacity (i.e., workforce), and process (services provided to ASD patients, partnerships) (Aluttis et al. 2014; Handler et al. 2001) and on the basis of national and international recommendations for ASD. As shown in Table 2, the questionnaire consisted of three parts and comprised both open- and closed-ended questions.

Since the country-specific context represents one of the core domains for public health capacity (Aluttis et al. 2014), data on regional policies concerning ASD were also collected for acquiring information about the "macrocontext" in which ASD health care is embedded in any single region. For this analysis, official websites of the Regional Health Systems were consulted in order to obtain information on regional ASD policies and legislation, specifically on actions taken by the Italian regions with respect to the following: (a) the adoption of IAAP (Conferenza Unificata 2012); (b) the release of formal recommendations for ASD's management; (c) the presence of funded regional ASD action plans; and (d) the presence of regional or local agreements defining pediatricians role and ASD early referral protocol. The survey of the regional ASD policies is updated to the year 2015 .

Table 2 Structure of the questionnaire: Section/Domain and topics included in the survey

\begin{tabular}{|c|c|c|}
\hline Section/Domain & Description & $\begin{array}{l}\text { n. of } \\
\text { items }\end{array}$ \\
\hline $\begin{array}{l}\text { I) Location and } \\
\text { typology of the } \\
\text { CAMHs }\end{array}$ & Address; Public/Private & 2 \\
\hline $\begin{array}{l}\text { II) Structural capacity } \\
\text { of the unit }\end{array}$ & $\begin{array}{l}\text { Intensity of care, Staff composition; } \\
\text { Working hours per week; Level of } \\
\text { use of digital technology }\end{array}$ & 5 \\
\hline III) Processes & $\begin{array}{l}\text { Percentage of human resources } \\
\text { allocated to ASD; ASD training } \\
\text { programs; Protocols and instruments } \\
\text { used for ASD patient diagnostic } \\
\text { assessment and characterization; } \\
\text { Available interventions; } \\
\text { Collaboration between agencies } \\
\text { (pediatricians, adult local public } \\
\text { services and schools) }\end{array}$ & 17 \\
\hline
\end{tabular}




\section{Data Analyses}

Data relative to closed-ended questions (with pre-coded response options) were analyzed descriptively using frequency distribution of items. Data relative to open-ended questions were analyzed using content analysis; recurring issues were listed and described using frequency distribution. The units participating to the survey were grouped based on their legal status in public and private facilities and according to the geographical macro-areas (North, South, Center and Islands) where the units are located in. These regional groupings are officially codified by the Italian National Institute of Statistics (ISTAT), which is a public research organization appointed for providing official statistics for Italy. The chi-square test was used to compare the distribution of categorical variables between public and private facilities, and among geographical macro-areas. The Fisher's exact probability test was used when requirements of the chi-square test concerning the expected frequencies were unmet. When a significant difference between public and private units was observed, comparisons among macro-areas were performed separately within public and private units. Bonferroni's correction was applied to pairwise post-hoc comparisons among macro-areas (6 comparisons when no difference was found between public and private units that were consequently analyzed together; 12 comparisons when public and private units differed and were consequently analyzed separately). Comparisons among subgroups of units with respect to the weekly working hours of health professionals (quantitative variable) were performed using the Brown-Forsythe analysis of variance (ANOVA) and the Welch $t$ test, to take into account possible heteroscedasticity among subgroups. Specifically, the Brown-Forsythe ANOVA was performed to compare the four macro-areas, and Welch's $t$ test to evaluate differences between public and private units. Pairwise post-hoc comparisons between macro-areas in the overall group of CAMHs or within public and private units separately were performed by Welch's $t$ test with Bonferroni's correction (accounting for 6 or 12 comparisons, respectively). Analyses were performed using STATA (Stata Statistical Software, Release 8.1. College Station, TX: Stata Corporation).

\section{Results}

\section{Intensity of Care}

In Italy, public vs private sectors were significantly different with respect to the proportion of units delivering outpatient services $\left(97.1 \%\right.$ vs $81.6 \%$, public vs private $\chi^{2}(1)=35.50$, $p<0.0001)$, day patient services $\left(16.4 \%\right.$ vs $3.5 \%, \chi^{2}(1)=$ $12.50, p=0.0004)$, semi-residential $\left(9.9 \%\right.$ vs $37.7 \%, \chi^{2}$ $(1)=50.17, p<0.0001)$, and residential services $(5.2 \%$ vs $\left.17.5 \%, \chi^{2}(1)=18.19, p<0.0001\right)$. Differences among geographical macro-areas were found within public CAMHs with respect to the proportion of units delivering semi-residential $\left(\chi^{2}(3)=18.79, p=0.0003\right)$ and residential services $\left(\chi^{2}(3)=\right.$ $19.69, p=0.0002$ ) (see Table 3 for post-hoc comparisons). No significant differences among geographical macro-areas were found for private units (all ps $>0.05$ ).

\section{Use of Digital Technology and Regional Health Information Exchange}

More than half (60.1\%) of the surveyed CAMHs reported the use of an electronic data system for collecting, storing, and retrieving clinical data, as well as data sharing with the general Regional Health Information System (68.2\%). No significant differences were found between public and private units for both these variables $\left(\chi^{2}(1)=0.97, p=0.3247 ; \chi^{2}(1)=1.93\right.$, $p=0.1648)$. By contrast, the use of digital technology resulted to be unevenly distributed across the Italian territory (use of an electronic data system: $\chi^{2}(3)=69.49, p<0.0001$; data sharing: $\left.\chi^{2}(3)=80.83, p<0.0001\right)$. In particular, CAMHs based in the North reported the largest use of an electronic data system (post-hoc comparisons: North $82.4 \%$ vs Center $49.3 \%$, South $50.5 \%$ and Islands $40.7 \%$; all ps $<0.01$ ) and data sharing with the Regional Health Information System (post-hoc comparisons: North $88.1 \%$ vs Center $67.1 \%$, South $37.9 \%$ and Islands 59.3\%; all ps $<0.01$ ). The smallest percentage of units reporting the adoption of electronic clinical data sharing was found in the South (post-hoc comparisons: South vs all other macro-areas, all ps $<0.05$ ).

\section{Availability and Qualification of Health Professionals}

Table 4 shows the average number of weekly working hours per CAMH per health professional category. As expected, health professionals distribute differently in public and private units. In particular, mean weekly working hours of therapists (Neurodevelopmental Disorders Therapists, Speech Therapists and Educators) were larger in private than in public CAMHs (95.6 vs. 27.3, Welch's $t=4.55, \mathrm{df}=92, p<0.0001$; 127.4 vs. 62.6 , Welch's $t=3.98, \mathrm{df}=101, p<0.0001 ; 102.8$ vs. 19.3, Welch's $t=4.85, \mathrm{df}=92, p<0.0001$ respectively), while mean weekly working hours of Child and Adolescent Psychiatrists were larger in public than in private CAMHs (56.70 vs 33.40, Welch's $t=4.13, \mathrm{df}=210, p<0.0001$ ). Within public units, territorial heterogeneity was observed in mean weekly working hours of Child Adolescent Psychiatrists, Psychologists, Neurodevelopmental Disorders Therapists, Speech Therapists, Educators and Social workers (Brown-Forsythe ANOVA, all ps $<0.0003$ ). Specifically, the availability of human resources (Child Adolescent Psychiatrists, Psychologists, Neurodevelopmental Disorders Therapists and Speech Therapists) was higher in CAMHs 
Table 3 Percentage of public and private CAMHs across categories of intensity of care, split by macro-area

\begin{tabular}{lcllll}
\hline \multirow{2}{*}{ CAMHs } & \multicolumn{2}{l}{ Intensity of care } & & \\
\cline { 2 - 5 } & Outpatient & Day-patient & Inpatient & Semi-residential & Residential \\
\hline Public & & & & $12.7^{\mathbf{a}}$ \\
North & 94.0 & 14.9 & 7.5 & $17.3^{\mathbf{a}}$ & $11.9^{\mathbf{c}}$ \\
Center & 97.1 & $10.6^{\mathbf{b}}$ & 3.8 & 5.0 & 2.9 \\
South & 100.0 & 28.3 & 3.3 & 0.0 & 0.0 \\
Islands & 100.0 & 17.2 & 14.9 & 9.9 & 5.1 \\
Italy & 97.1 & 16.4 & 7.5 & & \\
Private & & & & 25.9 & 19.4 \\
North & 66.7 & 7.4 & 3.7 & 44.4 & 12.5 \\
Center & 86.1 & 2.8 & 5.6 & 50.0 & 10.5 \\
South & 84.4 & 0.0 & 0.0 & 21.1 & 17.5 \\
Islands & 89.5 & 5.3 & 5.3 & 37.7 & \\
Italy & 81.6 & 3.5 & 3.5 & & \\
\hline
\end{tabular}

The $\chi^{2}$ test refers to the overall differences among macro-areas within public and private units for any intensity of care. Post-hoc comparisons: (a) $p<0.01$ vs Islands; (b) $p<0.05$ vs South; (c) $p<0.05$ vs Islands based in the North and Center in comparison with those in the South and Islands (all post-hoc comparisons ps $<0.05$ ). Moreover, CAMHs based in the Islands showed a lower availability of educators (Islands vs Center, $p<0.01$ ) and of social workers (Islands vs Center, $p<0.01$ ). When private unit were examined, the difference among macro-areas was significant only for neurodevelopmental disorders therapists, speech therapists, and educators (Brown-Forsythe ANOVA, $p=0.004$, 0.0068 , and 0.0243 , respectively), with lower availability of resources in Islands especially compared to the South $(p<0.05$; see Table 4 for more details).

\section{Proportion of ASD Patients and Working Time Allocated to ASD Patients}

To estimate a proxy of the average medical burden allocated to ASD by the surveyed CAMHs, we asked participants to indicate the percentage of ASD patients on the total number of patients served in their units, and the percentage of weekly working hours allocated to ASD patients (independently of the service provided, i.e., diagnosis or treatment). The reported percentages were classified in three classes: low proportion, $<30 \%$; medium proportion, $31-70 \%$; high proportion, $>$
Table 4 Average number of weekly working hours of each health professional category per CAMH unit, split by macro-area

\begin{tabular}{|c|c|c|c|c|c|c|c|c|}
\hline \multirow[t]{2}{*}{ CAMHs } & \multicolumn{8}{|c|}{ Average number of weekly working hours per CAMH unit } \\
\hline & CAP & PSY & NDT & ST & EDU & SW & NUR & PSYC \\
\hline \multicolumn{9}{|l|}{ Public } \\
\hline North & $70.5^{\mathrm{ab}}$ & $95.1^{\mathrm{ab}}$ & $35.3^{\mathrm{ab}}$ & $85.1^{\mathrm{ab}}$ & $17.3^{\mathrm{b}}$ & 17.4 & 20.5 & 0.5 \\
\hline Center & $64.5^{\mathrm{ad}}$ & $78.8^{\mathrm{ab}}$ & $37.9^{\mathrm{ab}}$ & $89.9^{\mathrm{ab}}$ & $20.4^{\mathrm{b}}$ & $28.1^{\mathrm{b}}$ & 15.3 & 1.4 \\
\hline South & 36.6 & $45.8^{\mathrm{b}}$ & 14.2 & 28.1 & $45.1^{\mathrm{b}}$ & $33.2^{\mathrm{b}}$ & 13.1 & 2.0 \\
\hline Islands & 39.4 & 22.0 & 10.8 & 18.4 & 3.2 & 10.8 & 11.7 & 0.0 \\
\hline Italy & 56.7 & 66.9 & 27.3 & 62.6 & 19.3 & 21.2 & 16.0 & 0.9 \\
\hline \multicolumn{9}{|l|}{ Private } \\
\hline North & 41.2 & 86.1 & $54.4^{\mathrm{c}}$ & 80.8 & $171.3^{\mathrm{b}}$ & 15.2 & 64.4 & 0.6 \\
\hline Center & 36.5 & 85.3 & 80.2 & 142.8 & 93.8 & 18.1 & 21.8 & 2.5 \\
\hline South & $19.2^{\mathrm{d}}$ & $63.0^{\mathrm{d}}$ & $184.8^{\mathrm{b}}$ & $187.5^{\mathrm{d}}$ & 121.8 & 35.7 & 18.9 & 1.1 \\
\hline Islands & 41.5 & 26.9 & 29.2 & 58.6 & 16.3 & 25.1 & 31.8 & 0.6 \\
\hline Italy & 33.4 & 68.0 & 95.6 & 127.4 & 102.8 & 24.0 & 31.4 & 1.3 \\
\hline
\end{tabular}

CAP, child adolescent psychiatrist; PSY, psychologist; NDT, neurodevelopmental disorders therapist; ST, speech therapist; EDU, educator; SW, social workers; NUR, nurse; PSYC, psychiatrist

Post-hoc comparisons (a) $p<0.01$ vs South; (b) $p<0.01$ vs Islands; c) $\mathrm{p}<0.05$ vs South; (d) $p<0.05$ vs Islands 
$70 \%$. The difference between public and private CAMHs was significant for both the variables (ASD patients: $\chi^{2}(2)=$ 22.57, $p<0.0001$; weekly working hours dedicated to ASD patients: $\left.\chi^{2}(2)=22.96, p<0.0001\right)$, with public units reporting low burden more frequently than private units (Table 5). Within public units, no significant differences among macro-areas were observed as for percentage of ASD patients (Fisher's exact probability test $p=0.058$ ). A significant difference across macro-areas was observed with respect to the percentage of weekly working hours dedicated to ASD patients (Fisher's exact probability test $p=0.002$ ), in particular between CAHMs located in the Center and in the South of Italy ( $p<0.05$ at post-hoc comparisons). In particular, about $11 \%$ of CAMHs based in the South reported a high proportion of weekly working hours vs $0 \%$ of CAMHs in the Center. Within private units, no significant differences were

Table 5 Percentage of public and private CAMHs reporting low, medium, and high proportion of ASD patients and professionals' weekly working hours dedicated to ASD patients, split by macro-area

\begin{tabular}{llll}
\hline CAMHs & Proportion of ASD patients & \\
\cline { 2 - 3 } & Low & Medium & High \\
\hline
\end{tabular}

\begin{tabular}{lccc}
\hline Public & & & \\
North & 96.5 & 0.7 & 2.8 \\
Center & 96.1 & 3.9 & 0.0 \\
South & 92.1 & 6.3 & 1.6 \\
Islands & 97.7 & 1.1 & 1.1 \\
Italy & 96.0 & 2.5 & 1.5 \\
Private & & & \\
North & 66.7 & 20.8 & 12.5 \\
Center & 75.7 & 5.4 & 18.9 \\
South & 56.3 & 21.9 & 21.9 \\
Islands & 76.2 & 19.0 & 4.8 \\
Italy & 68.4 & 15.8 & 15.8
\end{tabular}

CAMHs Proportion of weekly working hours dedicated to ASD patients

\begin{tabular}{lccc} 
& Low & Medium & High \\
Public & & & \\
North & 93.1 & 4.9 & 2.1 \\
Center & 88.3 & 11.7 & 0.0 \\
South & 81.0 & 7.9 & 11.1 \\
Islands & 89.7 & 4.6 & 5.7 \\
Italy & 89.2 & 7.1 & 3.8 \\
Private & & & \\
North & 50.0 & 25.0 & 25.0 \\
Center & 62.2 & 18.9 & 18.9 \\
South & 50.0 & 25.0 & 25.0 \\
Islands & 66.7 & 28.6 & 4.8 \\
Italy & 57.0 & 23.7 & 19.3 \\
\hline
\end{tabular}

Low proportion, $\leq 30 \%$; Medium proportion, $31-70 \%$; High proportion, $>70 \%$ found for both variables (Fisher's exact probability test $p=$ 0.209 and 0.514 , for percentage of ASD patients and percentage of time dedicated to ASD patients, respectively).

\section{Autism Training for Health Professionals}

The majority of the CAMHs surveyed reported to provide autism training to personnel working in the unit $(76.6 \%)$ and/or to offer financial support for the specialist training of their staff (56.7\%). We did not observe significant differences between public and private CAMHs with respect to both variables (staff training: $\chi^{2}(1)=1.73, p=0.1888$; financial support for training: $\left.\chi^{2}(1)=1.16, p=0.2823\right)$. The survey revealed territorial heterogeneity with respect to direct provision of training on autism $\left(\chi^{2}(3)=16.5, p=0.0009\right)$. In particular, a significantly higher percentage of CAMHs based in the North $(84.9 \%)$, in comparison with those in the Center $(72.1 \%)$ and in the South $(65.3 \%)$, reported to offer autism training to their staff ( $p<0.05$ and $p<0.01$, respectively). Instead, no significant difference was observed in comparison to Islands $(75.9 \%)$. Financial support for specialist training also resulted to be unevenly distributed across the territory $\left(\chi^{2}(3)=81.9, p<0.0001\right)$, with the highest percentage of CAMHs offering financial support in the North in comparison to the other macro-areas (North $81.7 \%$ vs. Center $49.3 \%$, South $47.4 \%$, and Islands $31.5 \%$, all ps $<0.01$ ).

\section{Diagnosis and Intervention Services for Children with ASD}

As for provision of diagnosis and intervention, a significantly higher percentage of public CAMHs reported to provide diagnosis than private units $\left(\chi^{2}(1)=129.4, p<0.0001\right)$. By contrast, intervention was more frequently reported by private than public units $\left(\chi^{2}(1)=15.4, p<0.0001\right)$. A significant difference among macro-areas was observed both in public and private CAMHs, either for diagnosis or interventions services (Fisher's exact probability test: public $p=0.0003$ for both diagnosis and intervention; private $p=0.0345$ for diagnosis and $p=0.0002$ for intervention). As for public CAMHs, a significant higher number of units located in the North reported to provide diagnosis compared to units based in Center $(p<0.01)$ and interventions compared to units in the South $(p<0.01)$. As for private units, a higher percentage of units based in the Center reported to provide intervention than units based in the Islands $(p<0.05)$ (see Table 6).

\section{ASD Diagnostic Assessment and Patient Characterization}

The provision of ASD diagnostic assessment and characterization was analyzed exclusively in the public units, as the 
Table 6 Percentage of public and private CAMHs providing diagnosis and intervention services for children with ASD, split by macro-area

\begin{tabular}{lcc}
\hline \multirow{2}{*}{ CAMHs } & \multicolumn{2}{c}{$\%$ of CAMHs providing } \\
\cline { 2 - 3 } & Diagnosis & Intervention \\
\hline Public & & \\
North & $100.0^{\mathrm{a}}$ & $83.9^{\mathrm{b}}$ \\
Center & 91.5 & 79.8 \\
South & 100.0 & 57.9 \\
Islands & 97.6 & 67.9 \\
Italy & 97.4 & 75.6 \\
Private & & \\
North & 75.0 & 100.0 \\
Center & 60.0 & $100.0^{\mathrm{c}}$ \\
South & 34.6 & 100.0 \\
Islands & 70.6 & 70.6 \\
Italy & 57.3 & 94.4 \\
\hline
\end{tabular}

Post-hoc comparisons: (a) $p<0.01$ vs Center; (b) $p<0.01$ vs South; (c) $p<0.05$ vs Islands

overall number of private CAMHs reporting to provide diagnosis was very low $(n=51)$.

As for diagnostic system, the majority (81.2\%) of respondent public units reported using the diagnostic system ICD10 , while the use of the DSM-IV was reported by $23.2 \%$ of the surveyed CAMHs. Heterogeneity among macro-areas was found for the use of ICD-10 $\left(\chi^{2}(3)=48.53, p<0.0001\right)$ and DSM-IV $\left(\chi^{2}(3)=53.64, p<0.0001\right)$. In particular, CAMHs based in the North reported the use of ICD-10 more frequently than units based in the other macro-areas (North $96.2 \%$ vs Center $73.3 \%$, South $82.3 \%$, and Islands $62.1 \%$, all ps < 0.05 ), while the use of DSM-IV was more frequently reported in the South $(38.7 \%)$ and Islands $(46.0 \%)$ than in the North $(9.4 \%)$ and Center $(15.8 \%)$ (all ps $<0.05)$. As for the presence of a formalized protocol for the diagnosis of ASD in the unit, $62.2 \%$ of the respondents reported the adoption of agreed standard operating procedures in their practice. Data highlight a territorial heterogeneity $\left(\chi^{2}(3)=35.5, p<0.0001\right)$, specifically a higher percentage of CAMHs adopting a formalized diagnostic protocol in the North than in the other macro-areas (North $78.0 \%$ vs Center $60.6 \%$, South $45.6 \%$, and Islands $47.4 \%$; all ps $<0.01$ ).

The majority of surveyed public units (96.3\%) reported the use of standardized diagnostic tools. The use of the Autism Diagnostic Observation Schedule-Generic (ADOS-G; Lord et al. 2001) was reported by $62.5 \%$ of the public units, while the use of the Autism Diagnostic Interview-Revised (ADI-R; Lord et al. 1994) was reported by $48.5 \%$ of the units (Fig. 2). The other most commonly used measures were the Childhood Autism Rating Scale (CARS; Schopler et al. 1988) used by $67.0 \%$ of the units, the Gilliam Autism Rating Scale (GARS; Gilliam 1995) used by $22.3 \%$ of the units, and the Autism Behavior Checklist (ABC; Krug et al. 1993), the use of which

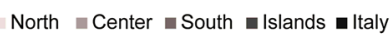

a ASD

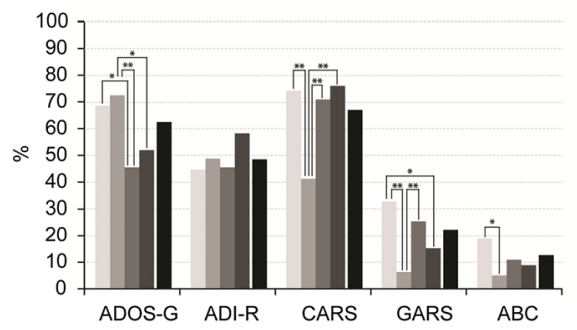

b Cognitive abilities

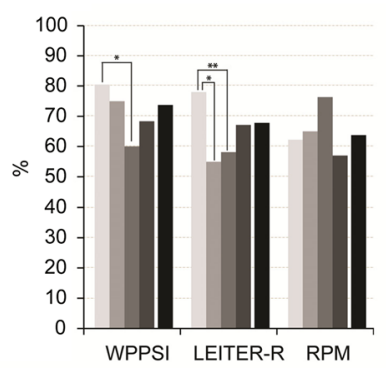

C Language

d Behavioral problems

e Adaptive behaviorl Global functioning
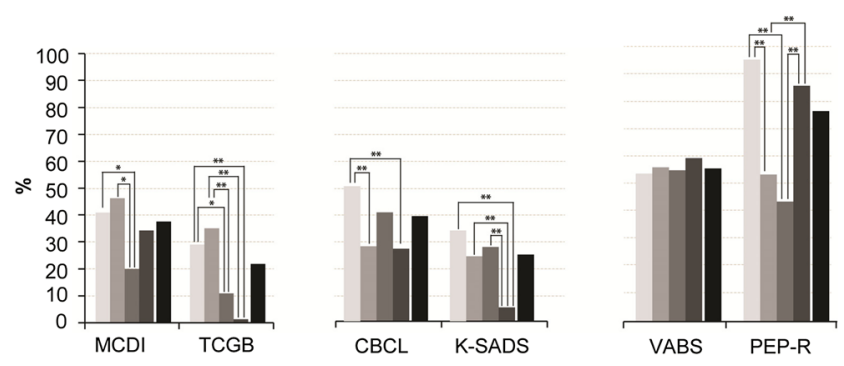

Fig. 2 Percentage of CAMHs reporting to use standardized tools for: a ASD assessment, b cognitive assessment; c language assessment; d assessment of behavioral problems; e adaptive behavior/global assessment of functioning. Post-hoc comparisons among macro-areas: $* p<0.05, * * p<0.01$

was reported by $12.6 \%$ of the surveyed units. As regards patient characterization, the survey considered four main areas of assessment: cognitive, language, adaptive behavior/global measures of functioning, and comorbid behavioral problems. Concerning the assessment of cognitive functions, the standardized tools more frequently reported in the surveyed CAMHs were: the Wechsler Preschool and Primary Scale of Intelligence (WPPSI; Wechsler 2012), reported by $73.7 \%$ of the units; the Leiter International Performance Scale-Revised (LEITER-R; Roid and Miller 1997), used by $67.8 \%$ of the units; the Raven's Progressive Matrices (RPM, Raven et al. 2000 ), used by $63.8 \%$ of the CAMHs. With regard to language assessment, less than half of the respondents (37.5\%) reported using the MacArthur-Bates Communicative Development Inventories (MCDI; Fenson et al. 2007), while the use of Grammar Comprehension Test for Children (TCGB; Chilosi and Cipriani 1995) was reported by $21.7 \%$ of the surveyed CAMHs. 
About half (53.4\%) of the surveyed CAMHs reported to assess adaptive behavior using the Vineland Adaptive Behavioural Scales (VABS; Sparrow et al. 2005), while $73.5 \%$ of units reported the use of the Psychoeducational Profile-Revised (PEP-R; Schopler et al. 1990) as a global measure of functioning and psychoeducative profiling. The Child Behaviour Checklist (CBCL; Achenbach and Edelbrock 1983) and the Schedule for Affective Disorders and Schizophrenia for School-Age Children (K-SADS; Kaufman et al. 1996) were the two most commonly used tools for assessing comorbid behavioral problems $(38.6 \%$ and $24.4 \%$, respectively). Statistical differences among macroareas in the use of standardized tools was reported for: $\operatorname{ADOS}-\mathrm{G}\left(\chi^{2}(3)=16.5, p=0.0009\right), \operatorname{CARS}\left(\chi^{2}(3)=31.0\right.$, $p<0.0001)$, GARS $\left(\chi^{2}(3)=24.5, p<0.0001\right), \operatorname{ABC}\left(\chi^{2}\right.$ $(3)=11.0, p=0.0117)$, WPPSI $\left(\chi^{2}(3)=10.4, p=0.0155\right)$, LEITER-R $\left(\chi^{2}(3)=15.9, p=0.0012\right)$, MCDI $\left(\chi^{2}(3)=10.9\right.$, $p=0.0123)$, TCGB $\left(\chi^{2}(3)=36.4, p<0.0001\right), \operatorname{CBCL}\left(\chi^{2}\right.$ (3) $=17.3, p=0.0006)$, KSADS $\left(\chi^{2}(3)=23.2, p<0.0001\right)$, PEP-R $\left(\chi^{2}(3)=77.3, p<0.0001\right)$. Post-hoc comparisons are shown in Fig. 2. No differences among macro-areas were found in the use of ADI-R $\left(\chi^{2}(3)=5.5, p=0.1404\right)$, RPM $\left(\chi^{2}(3)=5.6, p=0.1345\right)$, and $\operatorname{VABS}\left(\chi^{2}(3)=0.6, p=\right.$ $0.8895)$.

\section{Intervention Services for ASD Patients}

Most of the CAMHs providing outpatient treatment services reported to serve patients in the overall $0-17$ years age range $(88.3 \%)$. Surveyed CAMHs reported to offer various intervention programs to ASD patients, selecting one or more items among the following: Augmentative and Alternative Communication (AAC, used by the $74.8 \%$ of the units), the Training and Education of Autistic and Related Communication Handicapped Children (TEACCH, 45.2\%), the Applied Behavioral Analysis (ABA, 29.2\%), and the Early Start Denver Model (ESDM, 24.9\%), the Thérapie d'Echange et Developpment (TED, 13.4\%), the Developmental, Individual Difference, Relationship-based (DIR, 8.7\%) as well as other (no autism-specific) interventions, such as Speech Therapy (ST, 84.2\%) and Psychomotor Therapy (PMT, 82.4\%), Expressive-motor rehabilitation (EMR, 54.1\%), Family support (FS, 64.3\%), and Cognitive therapy (CT, 60.9\%). To evaluate if CAMHs adopted more than one intervention strategy and in which combination, we first grouped interventions in the following categories: (i) specific for ASD, including TEACCH, ABA, ESDM, TED, DIR; (ii) specific for communication, AAC; (iii) not specific for ASD, including ST and PMT; (iv) other interventions, including CT, FS, and EMR. For each category, the CAMHs were classified as YES when they offered at least one of the interventions included in the category. Finally, we estimated the frequency of combinations in the CAMHs providing outpatient intervention to patients in the whole age range $0-17$ years $(88 \%)$. Table 7 presents the percentage of CAMHs offering the different combinations of interventions, according to the macro-area. Heterogeneity in the pattern of treatments offered was found across macro-areas $\left(\chi^{2}(12)=50.1\right.$, $p<0.0001$ ), and in particular between North and other macro-areas (all ps $<0.05$ ).

\section{Collaborative Network for Children with ASD}

Collaborative treatment plans between clinicians, family pediatricians, teachers, and adult health services are reported for the public CAMHs, which are appointed by INHS to take charge of children with ASD in collaboration with other public health, social, and educational agencies.

Most of the units $(72.1 \%)$ reported to collaborate with pediatricians for ASD care, but only $18.4 \%$ through a formalized agreement. The percentage of CAMHs reporting the implementation of a formalized agreement was higher in the North and Center than in the South and Islands (North 29.1\% and Center $23.9 \%$ vs. South $6.3 \%$ and Islands $5.6 \%$; post-hoc comparisons $p<0.05$ for all). A specific collaboration with school for children with ASD (i.e., school-based clinical interventions for children with autism and training/tutoring on ASD management for teachers) was reported by $59.3 \%$ of the CAMHs, $12.5 \%$ through a formalized agreement. Macroareas did not differ with respect to the percentage of public CAMHs collaborating with schools through a formalized protocol. A collaboration with adult local public services (i.e., adult mental health services and services for adults with disabilities) aimed at guaranteeing continuity of care for children and adolescent with ASD (i.e., specific interventions and tutoring to support/improve transition process) was reported by $56.1 \%$ of the surveyed CAMHs. Of them, only $27.9 \%$ referred to a formalized agreed protocol. The percentage of CAMHs reporting the implementation of a formalized agreement was higher in the North and Center than in the South and Islands (North $37.7 \%$ and Center $38.6 \%$ vs. South $2.2 \%$ and Islands 4.9\%; post-hoc comparisons $p<0.01$ for all).

\section{Regional ASD Policies}

Results show that 11 Italian regions out of 20 (55\%) have issued and released formal recommendations for ASD's management, and have adopted the IAAP. The majority of regions (70\%) have reported at least one ASD funded action plan relative to the period 2010-2015. In half of the regions, a formal agreement defining the role of pediatricians and ASD early referral protocol have been approved, although in only four of them the agreement has been implemented across the whole region. As for CAMHs' knowledge of-and 
Table 7 Percentage of CAMHs offering autism-specific and other intervention programs, split by macro-area

\begin{tabular}{|c|c|c|c|c|c|c|c|c|}
\hline \multicolumn{4}{|c|}{ Interventions offered by CAMHs } & \multicolumn{5}{|c|}{ CAMHs } \\
\hline $\begin{array}{l}\text { Specific } \\
\text { for ASD }\end{array}$ & $\begin{array}{l}\text { Specific for } \\
\text { communication }\end{array}$ & $\begin{array}{l}\text { Not specific } \\
\text { for ASD }\end{array}$ & $\begin{array}{l}\text { Other } \\
\text { interventions }\end{array}$ & North & Center & South & Islands & Italy \\
\hline Yes & Yes & Yes & Yes & 43.7 & 37.6 & 62.0 & 32.4 & 43.4 \\
\hline Yes & No & Yes & Yes & 3.4 & 6.9 & 6.0 & 20.6 & 7.4 \\
\hline No & Yes & Yes & Yes & 29.9 & 16.8 & 2.0 & 8.8 & 17.3 \\
\hline No & No & Yes & Yes & 3.4 & 23.8 & 16.0 & 8.8 & 14.0 \\
\hline \multicolumn{4}{|c|}{ Other combinations $\S$} & 19.5 & 14.9 & 14.0 & 29.4 & 18.0 \\
\hline
\end{tabular}

Specific for ASD. At least one of the following: TEACCH, Training and Education of Autistic and Related Communication Handicapped Children; ABA, Applied Behavioral Analysis; ESDM, Early Start Denver Model; TED, Thérapie d'Echange et Developpment; DIR, Developmental, Individual Difference, Relationshipbased

Specific for communication. AAC, Augmentative and Alternative Communication

Not-specific for ASD. ST, speech therapy; PMT, psychomotor therapy

Other interventions. CT, cognitive therapy; FS, family support; EMR, expressive-motor rehabilitation

$\S$ Including all the combinations that are adopted by less than 5\% of the CAMHs all over Italy involvement in - regional plans, about 50\% of the surveyed CAMHs reported to be aware of regional ASD policies; only $70 \%$ of these reported to have been involved in regional policies.

\section{Discussion}

In the present study, we provide information on the presence, distribution, and structural characteristics of CAMHs within the Italian territory, including intensity of care provided, staff composition, professionals' working hours per week, and level of use of digital technology. Since ASD is in charge of units that serve together neuropsychiatric, psychiatric, and neurological disorders, this information permits to evaluate the structural environment in which ASD services are provided. In addition, we describe CAMHs capacity to provide specific care to ASD patients, by means of surveying the processes for ASD management, included the use of protocols and instruments for diagnostic assessment, functional and clinical characterization, and treatment.

The distribution of expected CAMHs results to be consistent with the statutory organization of INHS, based on an extensive service network throughout the Italian territory. The widespread CAMHs distribution represents a good starting point for the INHS to comply with international recommendations for ASD, which stress the importance to deliver community-based services near to patients' home communities. However, our data suggest that the number of units in the South of Italy is undersized, considering the mean population $(0-17$ years) living in the CAMHs catchment areas. In addition, the surveyed public CAMHs count on no more than two units for professional group on average ( $36 \mathrm{~h}$ is the current weekly working hours for a full-time contract) to manage approximately $1500-3000$ children and adolescents in need of mental health services per CAMH (Frigerio et al. 2009; Pedrini et al. 2012; WHO 2018). The low availability of working hours/week goes with the low availability of onward and long-term support options reported by the CAMHs (13\%one of eight — has a day-care facility and $4 \%$ - one of 25 - has a long-care facility).

As for ASD, current available prevalence estimates in Italy originate from local sources, namely two regional administrative registers and one single population-based study, performed in one Italian province (Pisa, ASDEU 2016, age 79 years, 11.5/1000; Narzisi et al. 2018). Lacking an Italian national estimate of ASD prevalence, hardly we can assess the exact number of children and adolescents with ASD in need of services, which represents a limitation of the present study for the evaluation of CAMHs capacity to manage ASD patients. However, we can reasonably speculate that the low availability in terms of professionals' working hours/week and of onward and long-term support options observed in CAMHs impacts also on ASD users. Therefore, the present study appears to confirm the conclusions of previous surveys that highlighted the necessity of strengthening services' structural capacity in order to meet adequately the requirements of ASD patients, particularly those in need of high support (Rogers et al. 2016; Thabrew and Eggleston 2018).

Considerable variability across geographical macro-areas was found in the provision of diagnostic and intervention services. In particular, CAMHs in the South and partially in the Islands showed a service capacity lower than CAMHs in the North with respect to day-care and long-care facilities, availability of professionals, and provision of intervention services. CAMHs in the South reported also less use of digital technology for data collection and sharing, and a scarce implementation of agreed standard operating procedures for ASD. 
Moreover, the financial investment in staff training resulted very limited: only less than half of the interviewed units reported to offer financial support for specialist training, being Northern Italy the only exception. It is worthwhile recalling that financial support for in- and out-service training for ASD professional development is important and absolutely necessary to ensure a high quality workforce; actually, it has been considered a good indicator of care quality and one of the areas for improvement in ASD management (Thabrew and Eggleston 2018).

High variability across macro-areas was also reported when the use of instruments for ASD identification and children's functional assessment were implied. In particular, ADOS and CARS appear the most frequently used tools for ASD diagnosis by public CAMHs, followed by the ADI-R. These data are similar to those collected in a recent survey mapping measures used for ASD diagnostic patient assessment and characterization across European countries (EU-AIMS project network; Ashwood et al. 2015). ADOS and ADI are in fact the two most widely used ASD observation and interview measures across EU. Although there are evidence for the reliability and validity of these tools, they have the disadvantage to be costly, in terms of training, administration, and price (Ashwood et al. 2015; McConachie et al. 2015). The reported lower use of these instruments in the Southern Italy mirrors results obtained in the EU-AIMS clinical network, in which geographical areas with relatively lower resources (such as Eastern Europe) were reported to use ADOS and ADI the least, suggesting that economic disparity factors may be a potential barrier to diagnosis. Language and comorbid psychopathology measures (e.g., CBCL) are not extensively used in Italian public CAMHs (similarly to what observed in European sites, Ashwood et al. 2015), a result that could indicate a gap in knowledge on autism comorbid clinical signs, or a scarcity of reliable ASD-specific tools to investigate these functional domains in ASD (McConachie et al. 2015).

As for the implementation of interventions for ASD management, the survey highlights a trend towards the use of currently recommended approaches of intervention. Results show that most of the CAMHs offer more than one intervention strategy. However, further patient-based investigation will be necessary to understand if ASD patients in charge of a specific CAMH undergo similar intervention programs regardless of their characteristics (age, ASD severity, adaptive functioning, comorbidities), or if a selection of interventions chosen among those overall offered by the CAMHs are combined in individually tailored programs ("eclectic approach"; Odom et al. 2012; Wong et al. 2015). Notably, survey data on working hours per week reported by each CAMH evidenced a lower number of working hours/week per CAMH of professionals that usually are skilled to implement interventions (e.g., therapists) than professionals usually appointed to make diagnosis (i.e., psychiatrists and psychologists), especially in public CAMHs. This finding appears highly critical in the delivery of early and intensive psycho-educative or behavioral interventions, considered elective for ASD management.

The higher presence of psychiatrists and psychologists than therapists suggests a higher availability of diagnostic rather than of intervention services in public CAMH units. In Italy, public CAMHs should guarantee both diagnosis and intervention services to children and adolescents with neurodevelopmental disorders. Indeed, the present survey evidenced that about the $75 \%$ of public CAMHs reported to offer intervention services for ASD, with the lowest percentage reported in the South $(58 \%)$. By contrast, private units reported to offer diagnosis services, though these units are formally appointed to the implementation of individual rehabilitation programs only (Law 388/78, Art. 26). A more careful governance that clearly assigns the roles of public and private providers and monitors the provision of diagnosis and intervention services appears advisable.

Finally, the present survey shows that in Italy the implementation of a collaborative networking with schools, as well as with local adult mental health services and services for adults with disabilities, is still unsatisfactory for individuals with ASD, especially when considering the paucity of standard operating agreements that specify health-care workflow and professionals' roles. Italian as well as international guidelines agree that services should take a wide, varied, and adaptable approach, in order to allow people living as independently as possible. This strategy calls for integrated pathways of care able to target the formal and informal child and family social network, the educational agency, as well as transitions at all developmental stages from preschool through adulthood (Ministries of Health and Education New Zealand 2016; NICE 2012; SIGN 2016; SNLG 2011). Our results are in line with a large scale survey of the perceptions of professionals involved in ASD care in the UK (Rogers et al. 2016): although professionals interviewed reported to frequently liaise with other services (e.g., school, employer), they still considered services as being fragmented and disjointed and felt the need of streamlined support services for individuals with ASD and their families.

As a concluding remark, our results highlight the need of specific national programs to strengthen structural capacity in this specific health sector and to point out the unacceptable inequalities evidenced within the country. In Italy, the political asset of regions is based on a substantial autonomy: regions can decide autonomously how their health systems should be structured within a general framework established at national level. This type of organization has led to a broad regional variability and to some inequalities in terms of available resources and services, and thus to the lack of national harmonization (OECD 2017). Results from the present survey 
suggest that heterogeneity among Italian macro-areas are associated with variation in ASD regional policies in term of implementation of the IAAP. This is in line with a recent study that compared Canadian and UK policies governing ASD management (Penner et al. 2018). The study by Penner and colleagues showed that variation in ASD diagnostic assessment, including necessary tools and personnel, as well as provision of interventions, may result from federalist systems, while more centralized health-care systems facilitate the development of national standards and processes. In Italy, the recent IAAP points to the harmonization of ASD diagnostic and characterization measures, agreement on standard operating procedures, support for joint training programs and a continuous and adequate monitoring and evaluation of ASD health services. The present survey could represent a firsttier analysis useful to evaluate the impact of the Italian policies on ASD management. Further actions to be implemented should be: (i) monitoring the availability of long-term and daily-care facilities; (ii) a more careful planning of human resources in terms of their availability and training; (iii) the harmonization of standard diagnostic operating procedures for a more reliable evaluation of the actual number of children with a diagnosis of ASD; (iv) the promotion of services' collaboration with external agencies (e.g., school, social services) and strengthening of health service integration throughout transition into adulthood. At a social and political level, our study also confirms the need to contrast inequalities in service provision across and within geographical areas (Salomone et al. 2016), which asks for a proper political solution.

\section{Limitations}

Information provided by the present survey is not fully representative of all Italian services that identify and manage children with ASD. Furthermore, the present study does not allow speculating on factors that drive the differences in service provision we found across geographical macro-areas. One possible approach might be to collect a more detailed information on differences and similarities in regional health policies and specific funding for ASD programs. Furthermore, a patient-centered study could provide individual information on intervention strategies in order to verify clinical characteristics that justify the choice of a specific intervention or a combination of interventions. Finally, the current investigation did not survey patient' and/or family's perspectives on the characteristics of health services that are perceived as important and valued. Indeed, this aspect should be further investigated to attain a shared vision among professionals, families, and people on the autism spectrum for what services should be provided and how and potentially what services and interventions should be prioritized (McConachie et al. 2015).
Acknowledgements We would like to thank Dr. Teresa di Fiandra and Dr. Giovanna Romano (Directorate General for Prevention, Italian Ministry of Health) for their useful advices during questionnaire development and for critical reading of the manuscript. We are also very grateful to Dr. Antonella Gigantesco and Dr. Angelo Picardi (Center for Behavioral Sciences and Mental Health, Istituto Superiore di Sanità) for their collaboration during questionnaire development and to Dr. Gemma Calamandrei (Center for Behavioral Sciences and Mental Health, Istituto Superiore di Sanità) for her valuable contribution to the discussion of the data. A special acknowledgement is due to Eleonora Daniele (Life-inside ONLUS), who believed in the commitment of the Istituto Superiore di Sanità towards people with ASD. A special thank you also goes to the Italian Regional Directorate of Health and the CAMH contact points for their precious collaboration.

Authors' Contributions MB was involved in interpretation of results, assisted with the data analyses, and wrote the paper. VA collected and prepared thedata for the qualitative and quantitative analysis. DC implemented Moodle platform for collection of data. FC was the co-promoter of the study, involved in the design and preparation of the study protocol and in the analysis of data, and collaborated in the writing of the paper. $\mathrm{AV}$ is the main researcher of the study, involved in coordination, design, execution and interpretation of data, and writing the manuscript. All authors read and approved the final version of the manuscript.

Flavia Chiarotti and Aldina Venerosi contributed equally to this work.

Funding Information The survey was funded by the Istituto Superiore di Sanità. Life-inside ONLUS granted a fellowship to V. Ambrosio for data collection. F.I.A. Fondazione Italiana per l'Autismo granted a research fellowship to M. Borgi for data analysis and writing of the manuscript and contributed to publication fee.

\section{Compliance with Ethical Standards}

Conflict of Interest On behalf of all authors, the corresponding author states that there is no conflict of interest.

Open Access This article is distributed under the terms of the Creative Commons Attribution 4.0 International License (http:// creativecommons.org/licenses/by/4.0/), which permits unrestricted use, distribution, and reproduction in any medium, provided you give appropriate credit to the original author(s) and the source, provide a link to the Creative Commons license, and indicate if changes were made.

\section{References}

Achenbach, T., \& Edelbrock, C. (1983). Manual for the child behavior checklist and revised child behavior profile. Burlington: Queen City Printers.

Aluttis, C., den Broucke, S. V., Chiotan, C., Costongs, C., Michelsen, K., \& Brand, H. (2014). Public health and health promotion capacity at national and regional level: a review of conceptual frameworks. Journal of Public Health Research, 3(1), 199.

Ashwood, K. L., Buitelaar, J., Murphy, D., Spooren, W., \& Charman, T. (2015). European clinical network: autism spectrum disorder assessments and patient characterisation. European Child and Adolescent Psychiatry, 24(8), 985-995.

Buescher, A. V., Cidav, Z., Knapp, M., \& Mandell, D. S. (2014). Costs of autism spectrum disorders in the United Kingdom and the United States. JAMA Pediatrics, 168(8), 721-728.

Chan, K. K. S., Lam, C. B., Law, N. C. W., \& Cheung, R. Y. M. (2018). From child autistic symptoms to parental affective symptoms: a 
family process model. Research in Developmental Disabilities, 75, $22-31$.

Chilosi, A., \& Cipriani, P. (1995). TCGB-Test di comprensione grammaticale per bambini. In Pisa: Edizioni del Cerro.

Conferenza Unificata. (2012). Accordo tra il Governo, le Regioni e le Province autonome di Trento e Bolzano, le Province, $i$ Comuni e le Comunità montane sulle "Linee di indirizzo per la promozione ed il miglioramento delle qualità e dell'appropriatezza degli interventi assistenziali nel settore dei Disturbi pervasivi dello sviluppo (DPS), con particolare riferimento ai disturbi dello spettro autistico". Rome: Presidenza del Consiglio dei Ministri.

Conferenza Unificata. (2018). Intesa tra il Governo, le Regioni, le Province autonome di Trento e Bolzano e gli Enti locali sul documento recante "Aggiornamento delle linee di indirizzo per la promozione ed il miglioramento della qualità e dell'appropriatezza degli interventi assistenziali nei Disturbi dello Spettro Autistico". Rome: Presidenza del Consiglio dei Ministri.

Donabedian, A. (1986). Quality assurance in our health care system. Quality Assurance and Utilization Review, 1(1), 6-12.

Elsabbagh, M., Divan, G., Koh, Y. J., Kim, Y. S., Kauchali, S., Marcin, C., et al. (2012). Global prevalence of autism and other pervasive developmental disorders. Autism Research, 5(3), 160-179.

Fenson, L., Marchman, V., Thal, D., Dale, P., \& Reznick, J. (2007). MacArthur-Bates communicative development inventories: user's guide and technical manual. Baltimore, MD: Brookes.

Fernandez-Alcantara, M., Garcia-Caro, M. P., Perez-Marfil, M. N., Hueso-Montoro, C., Laynez-Rubio, C., \& Cruz-Quintana, F. (2016). Feelings of loss and grief in parents of children diagnosed with autism spectrum disorder (ASD). Research in Developmental Disabilities, 55, 312-321.

Frigerio, A., Rucci, P., Goodman, R., Ammaniti, M., Carlet, O., Cavolina, P., et al. (2009). Prevalence and correlates of mental disorders among adolescents in Italy: the PrISMA study. European Child and Adolescent Psychiatry, 18(4), 217-226.

Gilliam, J. (1995). Gilliam autism rating scale. Austin, TX: Pro-Ed.

Handler, A., Issel, M., \& Turnock, B. (2001). A conceptual framework to measure performance of the public health system. American Journal of Public Health, 91(8), 1235-1239.

Hodgetts, S., Zwaigenbaum, L., \& Nicholas, D. (2015). Profile and predictors of service needs for families of children with autism spectrum disorders. Autism, 19(6), 673-683.

Howlin, P., Moss, P., Savage, S., \& Rutter, M. (2013). Social outcomes in mid- to later adulthood among individuals diagnosed with autism and average nonverbal IQ as children. Journal of the American Academy of Child and Adolescent Psychiatry, 52(6), 572-581.

Kaufman, J., Birmaher, B., Brent, D., Rao, U., \& Ryan, N. (1996). The schedule for affective disorders and schizophrenia for school-age children. Pittsburgh: University of Pittsburgh Medical Center.

Krug, D., Arick, J., \& Almond, P. (1993). Autism screening instrument for educational planning. Austin: Pro-Ed.

Lai, W. W., Goh, T. J., Oei, T. P., \& Sung, M. (2015). Coping and well-being in parents of children with autism spectrum disorders (ASD). Journal of Autism and Developmental Disorders, 45(8), 2582-2593.

Lord, C., Elsabbagh, M., Baird, G., \& Veenstra-Vanderweele, J. (2018). Autism spectrum disorder. Lancet, 392(10146), 508-520.

Lord, C., Rutter, M., DiLavore, P., \& Risi, S. (2001). Autism diagnostic observation schedule. Los Angeles: Western Psychological Services.

Lord, C., Rutter, M., \& Le Couteur, A. (1994). Autism diagnostic interviewrevised: a revised version of a diagnostic interview for caregivers of individuals with possible pervasive developmental disorders. Journal of Autism and Developmental Disorders, 24(5), 659-685.

McConachie, H., Parr, J. R., Glod, M., Hanratty, J., Livingstone, N., Oono, I. P., et al. (2015). Systematic review of tools to measure outcomes for young children with autism spectrum disorder. Health Technology Assessment, 19(41), 1-506.
Ministries of Health and Education. (2016). New Zealand autism spectrum disorder guideline (2nd ed.). Wellington: Ministry of Health.

Narzisi, A., Posada, M., Barbieri, F., Chericoni, N., Ciuffolini, D., Pinzino, M., et al. (2018). Prevalence of autism Spectrum disorder in a large Italian catchment area: a school-based population study within the ASDEU project. Epidemiology and Psychiatric Sciences, 1-10.

NICE - National Institute for Health and Clinical Excellence (2011). Autism spectrum disorder in under 19s: recognition, referral and diagnosis. Clinical Guideline Number 128. NICE.

NICE-National Institute for Health and Clinical Excellence (2012). Autism. The NICE guideline on recognition, referral, diagnosis and management of adults on the autism spectrum. Clinical guideline number 142. NICE.

NICE - National Institute for Health and Clinical Excellence. (2013). Autism spectrum disorder in under 19s: support and management. Clinical guideline number 170. In NICE.

Odom, S., Hume, K., Boyd, B., \& Stabel, A. (2012). Moving beyond the intensive behavior treatment versus eclectic dichotomy: evidencebased and individualized programs for learners with ASD. Behavior Modification, 36(3), 270-297.

OECD - European Observatory on Health Systems and Policies (2017). Italy: country health profile 2017, State of Health in the EU. Brussels: OECD Publishing, Paris/European Observatory on Health Systems and Policies.

Pedrini, L., Colasurdo, G., Costa, S., Fabiani, M., Ferraresi, L., Franzoni, E., et al. (2012). The characteristics and activities of child and adolescent mental health services in Italy: a regional survey. BMC Psychiatry, $12,7$.

Penner, M., Anagnostou, E., Andoni, L., \& Ungar, W. (2018). Environmental scan of Canadian and UK policies for autism spectrum disorder diagnostic assessment. Paediatrics and Child Health, pxy107. https://doi.org/10.1093/pch/pxy107.

Picardi, A., Gigantesco, A., Tarolla, E., Stoppioni, V., Cerbo, R., Cremonte, M., et al. (2018). Parental burden and its correlates in families of children with autism spectrum disorder: a multicentre study with two comparison groups. Clinical Practice and Epidemiology in Mental Health, 14, 143-176.

Raven, J., Raven, J., \& Court, J. (2000). Manual for Raven's progressive matrices and vocabulary scales. Oxford: Oxford Psychologists Press.

Rogers, C. L., Goddard, L., Hill, E. L., Henry, L. A., \& Crane, L. (2016). Experiences of diagnosing autism spectrum disorder: a survey of professionals in the United Kingdom. Autism, 20(7), 820-831.

Roid, G., \& Miller, L. (1997). Leiter international performance scale revised: examiner's manual. Wood Dale, IL: Stoelting.

Salomone, E., Beranova, S., Bonnet-Brilhault, F., Briciet Lauritsen, M., Budisteanu, M., Buitelaar, J., et al. (2016). Use of early intervention for young children with autism spectrum disorder across Europe. Autism, 20(2), 233-249.

Schopler, E., Reichler, R., Bashford, A., Lansing, M., \& Marcus, L. (1990). Individualized assessment and treatment for autistic and developmentally disabled children. Psychoeducational profilerevised (PEP-R). Austin, TX: Pro-Ed.

Schopler, E., Reichler, R., \& Renner, B. (1988). The childhood autism rating scale (CARS). Los Angeles: Western Psychological Services.

SIGN - Scottish Intercollegiate Guidelines Network. (2016). Assessment, diagnosis and interventions for autism spectrum disorders (p. 145). Edinburgh: SIGN (SIGN publication no.

SNLG - Sistema Nazionale per le Linee Guida (2011). Il trattamento dei disturbi dello spettro autistico nei bambini e negli adolescenti. Linea Guida 21. Roma: Istituto Superiore di Sanità.

Sparrow, S., Cicchetti, D., \& Balla, D. (2005). Vineland Adaptive Behavior Scales (Vineland II) survey interview form/caregiver rating form (2nd ed.). Livonia: Pearson Assessments.

Stewart, M., McGillivray, J., Forbes, D., \& Austin, D. (2017). Parenting a child with an autism spectrum disorder: a review of parent mental health and its relationship to a trauma-based conceptualisation. Advances in Mental Health, 15(1), 4-14. 
Thabrew, H., \& Eggleston, M. (2018). Spectrum of care: current management of childhood autism spectrum disorder (ASD) in New Zealand. Australasian Psychiatry, 26(3), 294-298.

Turcotte, P., Mathew, M., Shea, L. L., Brusilovskiy, E., \& Nonnemacher, S. L. (2016). Service needs across the lifespan for individuals with autism. Journal of Autism and Developmental Disorders, 46(7), 2480-2489.

Wechsler, D. (2012). Wechsler preschool and primary scale of intelligence (4th ed.). San Antonio: The Psychological Corporation.

WHO - World Health Organization (2018). Child and adolescent mental health. Retrieved from https://www.who.int/mental health/ maternal-child/child_adolescent/en/.
Wong, C., Odom, S. L., Hume, K. A., Cox, A. W., Fettig, A., Kucharczyk, S., et al. (2015). Evidence-based practices for children, youth, and young adults with autism spectrum disorder: a comprehensive review. Journal of Autism and Developmental Disorders, 45(7), 1951-1966.

Publisher's Note Springer Nature remains neutral with regard to jurisdictional claims in published maps and institutional affiliations. 